\title{
REGISTROS DE MAMÍFEROS FÓSSEIS NO LAJEDO DE SOLEDADE, APODI, RIO GRANDE DO NORTE, BRASIL
}

\author{
KLEBERSON DE OLIVEIRA PORPINO \\ Departamento de Ciências Biológicas, UERN, Rua Professor Antônio Campos, s/n, 59633-010, Mossoró, RN,Brasil. \\ kporpino@bol.com.br \\ MARIA DE FÁTIMA CAVALCANTE FERREIRA DOS SANTOS \\ Museu Câmara Cascudo, UFRN, Avenida Sen. Salgado Filho, 1398, Tirol, Natal, RN, Brasil. mfatima@ufrnet.br \\ LÍLIAN PAGLARELLI BERGQVIST \\ Departamento de Geologia, UFRJ, 21941-901, Rio de Janeiro, RJ, Brasil. bergqvist@ufrj.br
}

\begin{abstract}
RESUMO - São registradas novas ocorrências de mamíferos pleistocênicos coletados no Lajedo de Soledade, em Apodi (RN). O material consiste em ossos pós-cranianos, dentes isolados, osteodermos de carapaça e fragmentos de dentário encontrados em associação. Foram identificados: Hippidion sp., Glyptodon sp., Panochthus greslebini, Eremotherium laurillardi, Holmesina paulacoutoi, Palaeolama major, Xenorhinotherium bahiense, Equus (Amerhippus) cf. neogaeus, Smilodon populator e Toxodontidae. Registrase, pela primeira vez, Arctotherium sp., Cerdocyon thous, Protocyon troglodites, Leopardus cf. L. tigrinus e Tolypeutes tricinctus em associação com restos da megafauna no Estado. As características ecológicas dos taxa sugerem a vigência de condições ambientais distintas da atual para o Pleistoceno final-Holoceno na região, incluindo áreas abertas de savana em associação com fisionomias mais fechadas.
\end{abstract}

Palavras-chave: Mamíferos, Pleistoceno superior-Holoceno, Rio Grande do Norte, Apodi.

ABSTRACT - FOSSIL MAMMAL RECORDS FROM LAJEDO DE SOLEDADE, APODI, RIO GRANDE DO NORTE, BRAZIL. New occurrences of fossil mammals from Lajedo de Soledade, Pleistocene of Rio Grande do Norte State are presented. The material was found associated and includes postcranial elements, isolated teeth, carapace scutes and dentary fragments. The taxa identified are: Hippidion sp., Glyptodon sp. Panochthus greslebini, Eremotherium laurillardi, Holmesina paulacoutoi, Palaeolama major, Xenorhinotherium bahiense, Equus (Amerhippus) cf.neogaeus, Smilodon populator and Toxodontidae. Arctotherium sp., Cerdocyon thous, Protocyon troglodites, Leopardus cf. L.tigrinus and Tolypeutes tricinctus are reported for the first time in association with the megafauna in this state. The ecological requirements of the taxa suggest the existence of environmental conditions differents from the current ones during the Late Pleistocene-Holocene in the region, including open grassland habitats associated with more closed environments.

Key words: Mammals, late Pleistocene-Holocene, Rio Grande do Norte, Apodi.

\section{INTRODUÇÃO}

O Lajedo de Soledade, situado no município do Apodi (Figura 1), na região oeste do Estado do Rio Grande do Norte, corresponde à maior exposição de rochas carbonáticas da Formação Jandaíra, Cretáceo da bacia Potiguar (Bagnoli, 1994). A atuação de intenso processo de carstificação sobre fraturas e falhas de direção preferencial $\mathrm{NE} / \mathrm{SW}$ e NW/SE, resultou na formação de ravinas e cavernas (Córdoba et al., 1994). Estas guardam, além de fósseis, importante sítio arqueológico onde predominam grafismos rupestres.

As primeiras referências a fósseis de vertebrados no Lajedo de Soledade foram feitas por Rosado (1957) e SouzaCunha (1966), através de achados esporádicos. Santos (2001) registrou fósseis quaternários no Lajedo. Santos et al. (2002a) divulgaram novas informações sobre a associação faunística daquela localidade, com uma breve abordagem tafonômica, dando ênfase ao conteúdo de uma das ravinas: a Ravina do Leon (Figura 1). 

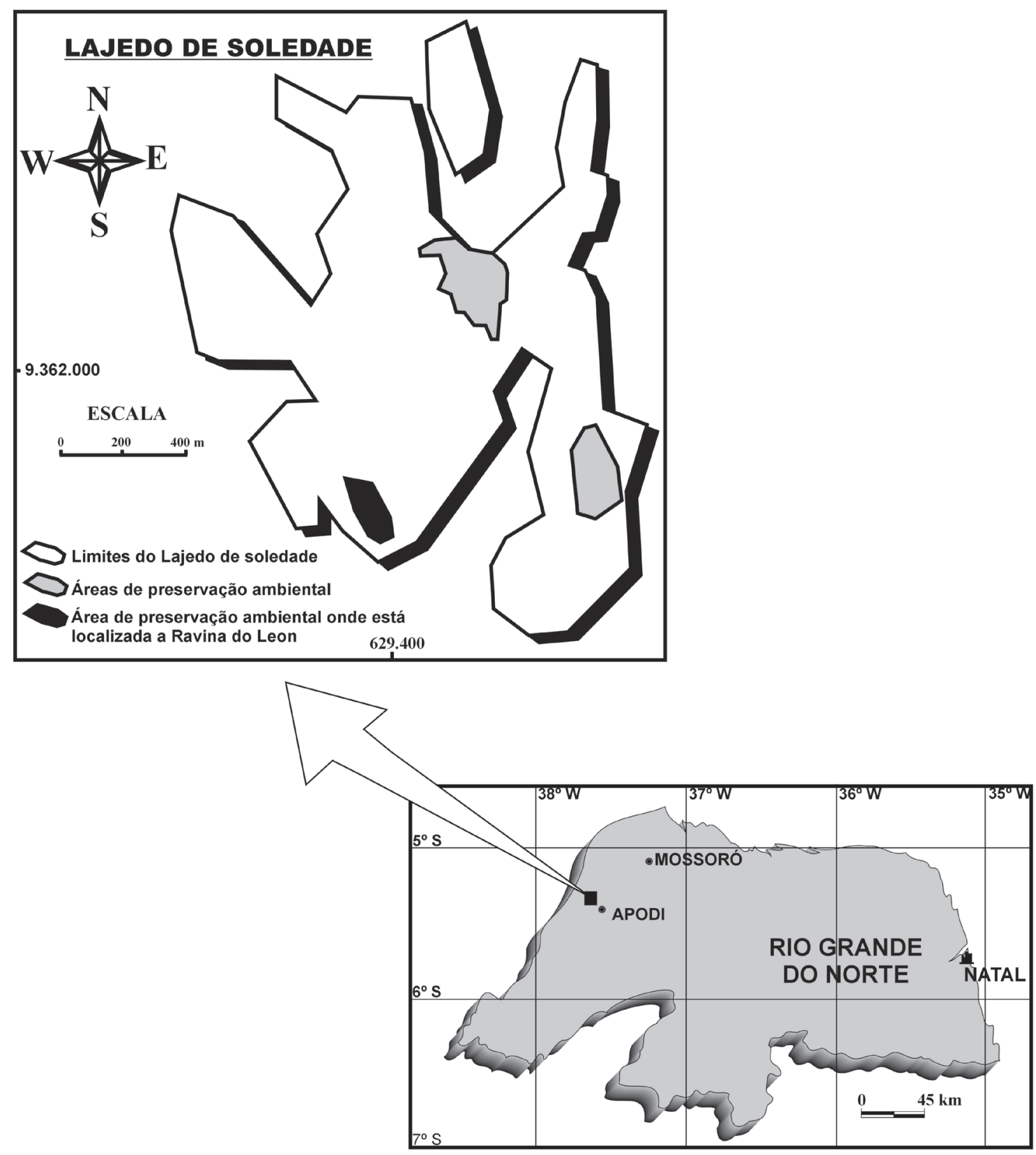

Figura 1. Mapa de localização do Lajedo de Soledade no Rio Grande do Norte, Brasil (modificado de Córdoba et al., 1994 e Santos et al., 2002a).

Figure 1. Location map of the Lajedo de Soledade in Rio Grande do Norte State, Brazil (modified from Córdoba et al., 1994 and Santos et al., 2002a).

O preenchimento sedimentar das ravinas se deu a partir de fonte próxima sob condições de pouco transporte, como atesta o baixo grau de arredondamento dos grãos de quartzo e dos fragmentos ósseos (Santos et al., 2002b). A preservação de pequenas peças e o grande número de pequenos fragmentos na parte mais profunda da ravina é um indicativo de que o material foi levado para a fenda quando esta se encontrava em incipiente estágio de desenvolvimento, ocasião em que a probabilidade de retenção de elementos esqueletais de maior tamanho seria menor.
Quanto à diagênese, o estudo de lâminas delgadas permitiu a verificação, nos fósseis, de processo de permineralização por material variado e substituição por mineral opaco, provavelmente limonita, de larga ocorrência ao longo do Lajedo (Santos, 2001).

Neste trabalho é feita uma identificação taxonômica mais precisa dos grupos constituintes da associação faunística do Lajedo de Soledade, com breves considerações paleoecológicas. Pela presença de Eremotherium laurillardi e Panochthus greslebini na associação local, considera-se o 
material coletado como pertencente ao Pleistoceno finalHoloceno.

O material estudado pertence ao Museu do Lajedo de Soledade (MLS), município de Apodi, e consiste de pequenas peças e fragmentos de ossos pós-cranianos, dentes e fragmentos de dentes de animais de grande, médio e pequeno porte. Em alguns casos, o material está muito danificado e a identificação do grupo foi feita graças a características bastante diagnósticas. As peças, inclusive aquelas pertencentes a animais com representação na fauna atual, apresentam coloração marrom em decorrência do mineral opaco predominante na área, e algumas formam pequenas concreções ferruginosas.

\section{PALEONTOLOGIA SISTEMÁTICA}

Ordem CARNIVORA Bowdich, 1821

Subordem CANIFORMIA Kretzoi,1943

Superfamília CANOIDEA Simpson, 1831

Família CANIDAE Fischer, 1817

Subfamília CANINAE Fischer, 1817
Cerdocyon thous Smith, 1839

(Figura 2A)

Material. MLS 01, $\mathrm{M}^{1}$ direito; MLS 02, fragmento de dentário esquerdo com $\mathrm{P}_{4}$ implantado; MLS 03, metade distal de rádio direito; MLS 04-05, falanges proximais; MLS 06, calcâneo esquerdo; MLS 07, astrágalo esquerdo.

Comentários. No Brasil, restos de $C$. thous foram coletados nas grutas calcárias de Minas Gerais em associação com a megafauna estudada por Lund (Lessa et al., 1999) e posteriormente por Cartelle \& Hartiwig (1996). Guérin et al. (1993) registram o táxon no sítio arqueológico de São Raimundo Nonato/Piauí, sob as mesmas condições de associação. Lessa et al. (1999) identificaram o táxon para o Pleistoceno finalHoloceno da Bahia. Atualmente, a espécie se distribui no Suriname, Guiana, desde a Colômbia e sul da Venezuela até o Paraguai, Uruguai, norte da Argentina e na maior parte do Brasil (Nowak, 1991), vivendo em matas, proximidades de matas ou em áreas abertas (Berta, 1982). As peças MLS 02, 03,04, 05, 06 e 07 são concordantes com os homólogos da espécie recente de $C$. thous. $\mathrm{O} \mathrm{M}^{1}$ (MLS 01) apresenta cíngulo
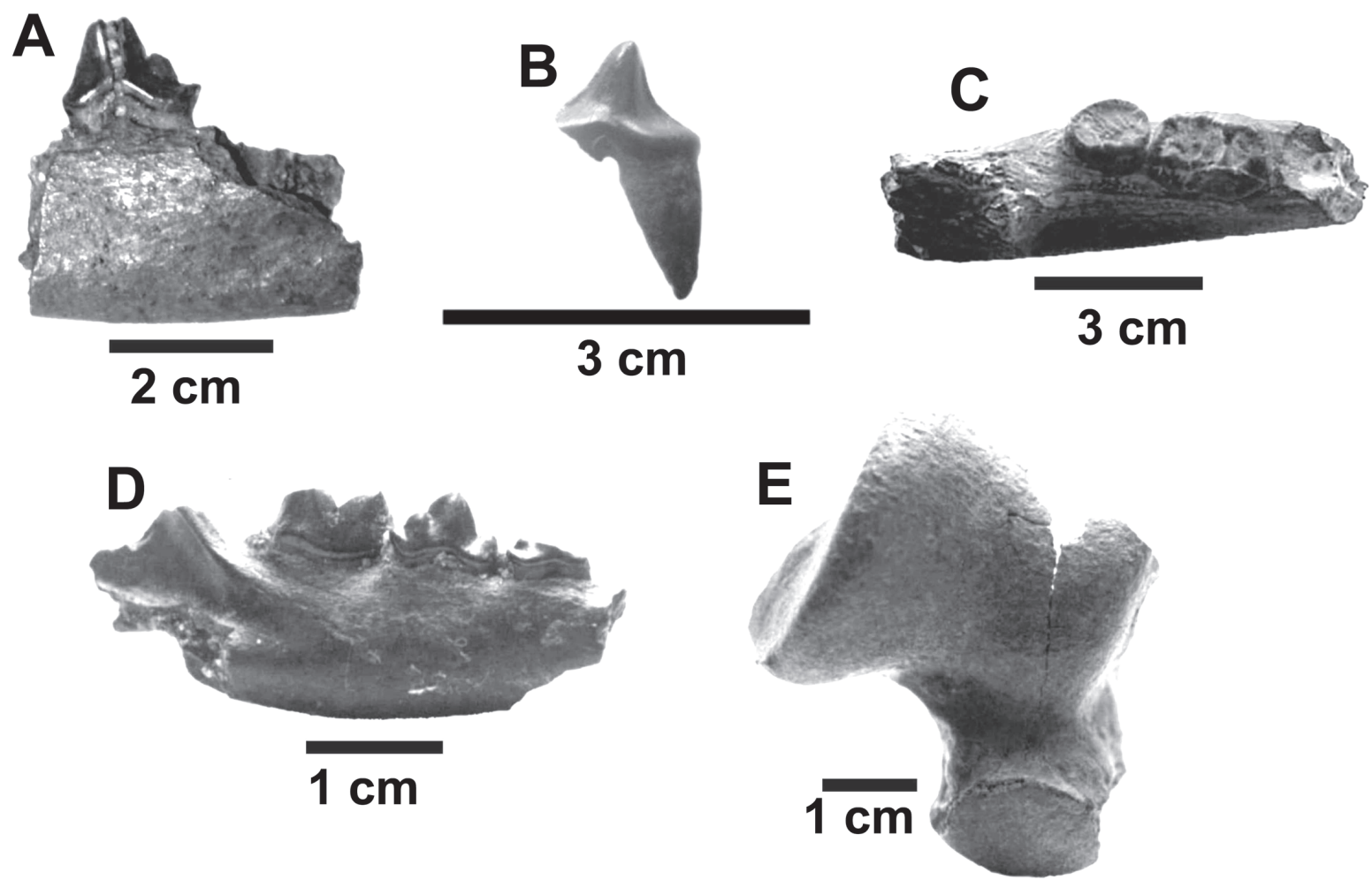

Figura 2. A. Cerdocyon thous, MLS 02, fragmento de dentário com $\mathrm{P}_{4}$ implantado em vista labial; B. Protocyon troglodites, MLS 08, $\mathrm{P}_{2}$ direito em vista lingual; C. Arctotherium sp., MLS 11, fragmento de dentário direito com $\mathrm{M}_{2}$ e $\mathrm{M}_{3}$ implantados; D. Leopardus cf. L.tigrinus, MLS 09, fragmento de dentário com $\mathrm{P}_{3} \mathrm{P}_{4}$ e $\mathrm{M}_{1}$ implantados em vista labial; E. Smilodon populator, MLS 10, astrágalo direito em vista dorsal. Figuras B, D, e E modificadas de Santos et al. (2002a).

Figure 2. A. Cerdocyon thous, MLS 02, dentary fragment with $\mathrm{P}_{4}$ in labial view; $\mathbf{B}$. Protocyon troglodites, $M L S$, 08 , right $\mathrm{P}_{2}$ in lingual view; C. Arctotherium sp., MLS 11, right dentary fragment with $M_{2}$ e $M_{3}$; D. Leopardus cf. L.tigrinus, MLS 09, dentary fragment with $P_{3} P_{4}$ e $M_{1}$ in labial view; E. Smilodon populator, MLS 10, right astragalus in dorsal view. Figures B, D e E modified from Santos et al. (2002a). 
bem desenvolvido por todo perímetro da coroa e hipocone bem marcado conforme a diagnose de Berta (1982) para a espécie.

\section{Protocyon troglodites (Lund)}

(Figura 2B)

Material. MLS 08, $\mathrm{P}_{2}$ direito; MLS 53, porção distal de rádio direito.

Comentários. Os achados originais desta espécie de canídeo extinto se devem a Lund (Cartelle \& Langguth, 1999). Registros posteriores de restos incompletos foram realizados por Paula-Couto (1979), Gomide (1989), Guérin et al. (1993) e Bergqvist (1995). Posteriormente, Cartelle \& Langguth (1999) estudaram material mais completo, incluindo um esqueleto incompleto ainda articulado do município de Campo Formoso (Bahia). Born \& Sedor (2001) registram um crânio da referida espécie coletado no Estado do Paraná. O dente MLS 08 é birradiculado, com perímetro da coroa mais largo distal que mesialmente e sem cúspide acessória, concordando com as descrições de Cartelle \& Langguth (1999).

Família URSIDAE Fischer, 1817 Subfamília TREMARCTINAE Merrian \& Stock, 1925

Arctotherium Burmeister, 1879

(Figura 2C)

Material. MLS 11, fragmento de dentário direito com $\mathrm{M}_{2} \mathrm{e}$ M implantados; MLS 12, $\mathrm{M}^{2}$ esquerdo isolado.

Comentários. São raros os achados de Ursidae no Brasil. Destacam-se os estudos de Paula-Couto (1960) sobre peças coletadas na região de Lagoa Santa, Minas Gerais, Trajano \& Ferrarezzi (1994) com material oriundo de Ubajara (CE), Cartelle (1998) e Lessa et al. (1999) com fósseis provenientes de grutas calcárias da Bahia e Ribeiro et al. (2003) no Rio Grande do Sul. No Rio Grande do Norte esta constitui a primeira ocorrência da família. Cartelle $(1994,1998)$ atribui os achados brasileiros a Arctotherium brasiliense (Lund). Entretanto, Soibelzon (2004), em uma revisão sistemática dos Tremarctinae fósseis sul-americanos, considera o dentário figurado em Cartelle (1994) e o material descrito em Cartelle (1998) como pertencentes a Arctotherium wingei Ameghino, 1902, enquanto o crânio figurado em Cartelle (1994) como possivelmente pretencente a Arctotherium vetustum Ameghino, 1885, apontando, desta maneira, para a possibilidade da ocorrência de mais de uma espécie para Arctotherium no Quaternário brasileiro. As características do $\mathrm{M}^{2}$ MLS 12 , entre as quais destaca-se a presença de lobo labial correspondente ao metacone pouco desenvolvido, ângulo mesial da coroa arredondado, cúspides linguais do trígono formando uma crista baixa e contínua, hipocone representado por uma crista mesiolingual e tálon muito inclinado línguo-distalmente formando uma superfície mastigatória contínua com o trígono, são concordantes com a morfologia de $A$. wingei, de acordo com Soibelzon (2004). Por outro lado, a presença de hipocone baixo e achatado e entoconido representado por três cúspides no $M_{2}$ e o $M_{3}$ com débil estreitamento do bordo labial entre o protoconido e hipoconido coincidem com as descrições de Soibelzon (2004) para A. vetustum. Todavia, considera-se necessário material mais completo para atribuições específicas mais consistentes.

Subordem FELIFORMIA Kretzoi, 1945

Superfamília FELOIDEA Simpson, 1931

Família FELIDAE Fischer, 1817

Subfamília FELINAE Fischer, 1817

\section{Leopardus cf. L. tigrinus}

(Figura 2D)

Material. MLS 09, fragmento de dentário direito com parte do $\mathrm{P}_{3}, \mathrm{P}_{4}$ e $\mathrm{M}_{1}$ implantados.

Comentários. A espécie L. tigrinus apresenta atualmente ampla distribuição, incluindo países da América Central e América do Sul. No Brasil é apontada para a região sul (Oliveira, 1998), embora também ocorra no Nordeste (Paiva \& Campos, 1995). Cartelle (1999) faz referência à ocorrência desta espécie no Pleistoceno de Minas Gerais. O material coletado no Lajedo de Soledade é ligeiramente maior, embora concordante nas demais características morfológicas quando comparado ao espécime de L. tigrinus UFPB-795 do Laboratório de Citogenética de Mamíferos da Universidade Federal da Paraíba.

\section{Subfamília MACHAIRODONTINAE Gill, 1872}

\section{Smilodon populator Lund, 1842}

(Figura 2E)

Material. MLS 10, astrágalo direito fragmentado.

Comentários. A espécie $S$. populator foi proposta com base em material encontrado na região de Lagoa Santa (MG) (Gomide et al., 1987). Posteriormente revelou-se que este felídeo era comum tanto na América do Norte como na do Sul, onde é registrado desde o Plioceno Superior. Seus achados, principalmente os do final do Pleistoceno, são muito comuns no Equador, Bolívia, Argentina e Brasil. Cartelle \& Abuhid (1989) refutam a subdivisão da espécie S. populator em subespécies com base somente na diferença de tamanho, concluindo que a forma norte-americana e a sul americana seriam coespecíficas. O astrágalo MLS 10 é aqui atribuído a $S$. populator em virtude de suas dimensões avantajadas, que contrasta, visivelmente, com peças homólogas de outros Carnivora do Pleistoceno final-Holoceno. 
Ordem CINGULATA Illiger, 1811

Superfamília GLYPTODONTOIDEA Gray, 1869

Família GLYPTODONTIDAE Gray, 1869

Subfamília SCLEROCALYPTINAE Trouessart, 1898

Tribo PANOCHTHINI Castellanos, 1927

\section{Panochthus greslebini Castellanos, 1941.}

(Figura 3A)

Material. MLS 13-17, osteodermos de carapaça.

Comentários. No Nordeste do Brasil os Panochthini são representados por duas espécies endêmicas, Panochthus greslebini e P. jaguaribensis Moreira, 1965, diferenciadas pela ornamentação da carapaça e do tubo caudal (Porpino \& Bergqvist, 2002). No Rio Grande do Norte, achados referentes a estes táxons foram registrados por Oliveira et al. $(1982,1984)$ e Porpino \& Bergqvist (2002). Os osteodermos aqui referidos apresentam ornamentação composta por figurinhas de pequeno tamanho, distribuídas sem orientação preferencial e sem figura central maior, o que permite atribuí-los à espécie $P$. greslebini, conforme as descrições de Moreira (1971) e Porpino \& Bergqvist (2002).
Subfamília GLIPTODONTINAE Gray 1869

Glyptodon Owen, 1838

(Figura 3B)

Material. MLS 18, MLS19,osteodermos de carapaça. Comentários. No Brasil, os achados referentes ao gênero Glyptodon foram, na maior parte, atribuídos à espécie-tipo Glyptodon clavipes Owen, 1839 (vide Winge, 1915; Paula- Couto, 1983; Cartelle, 1992). Para o Rio Grande do Norte, considerável quantidade de osteodermos e material pós-craniano diverso, coletada no município de Baraúnas, possivelmente pertence à espécie Glyptodon reticulatus Owen, 1845 (Porpino, 2000), a qual é registrada com dúvidas para o Rio Grande do Sul (Oliveira, 1996). Os osteodermos coletados no Lajedo de Soledade exibem o padrão ornamental em forma de roseta característico do gênero, porém o estado fragmentado e o desgaste na superfície externa dificultam a atribuição específica.
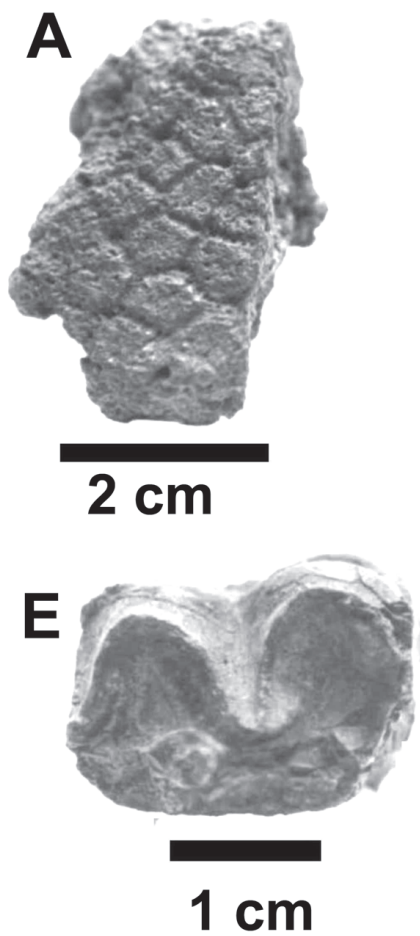
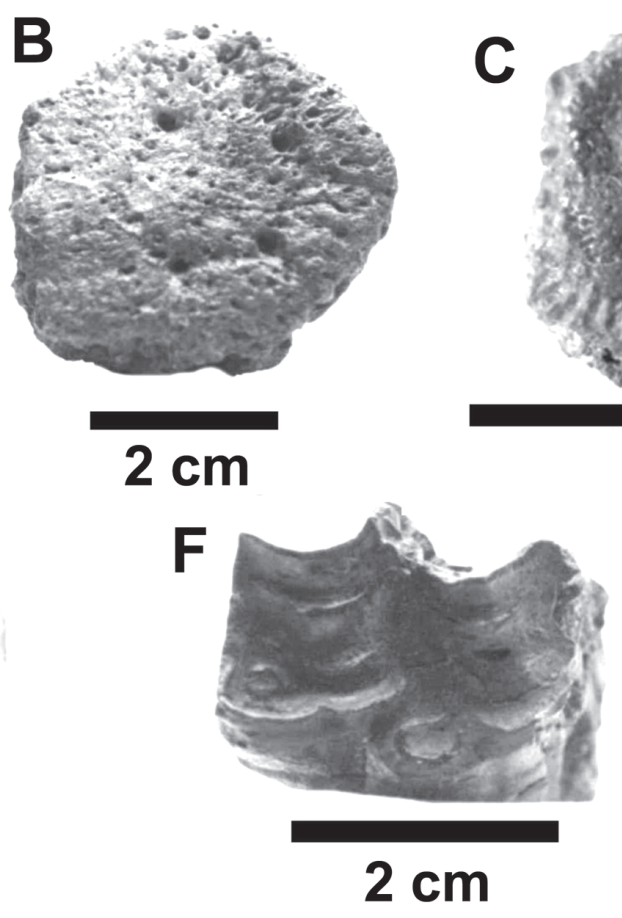
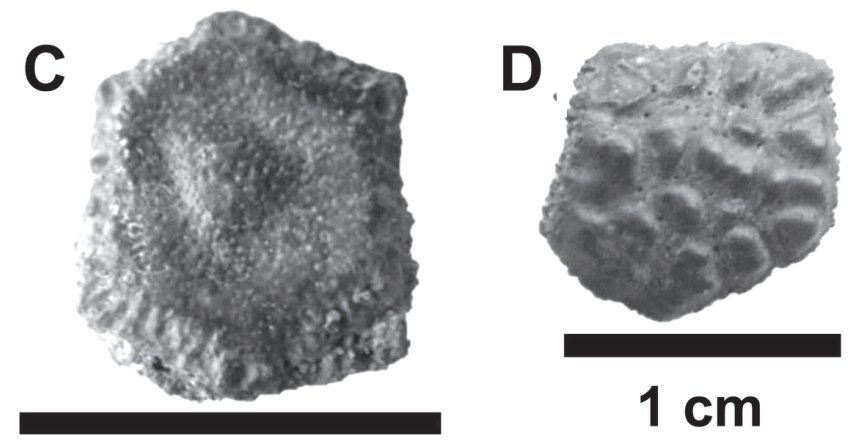

$3 \mathrm{~cm}$

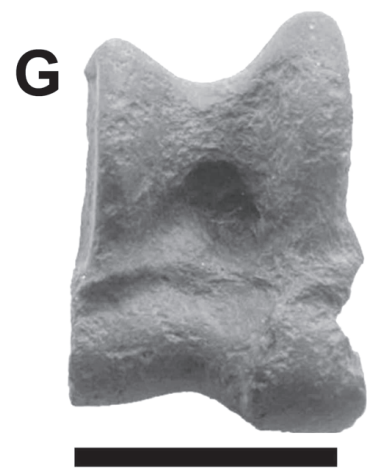

\section{$3 \mathrm{~cm}$}

Figura 3. A. Panochthus greslebini, MLS 13, fragmento de osteodermo; B. Glyptodon sp., MLS 18, osteodermo isolado; C. Holmesina paulacoutoi, MLS 22, osteodermo isolado; D. Tolypeutes tricinctus, MLS 20, osteodermo isolado; E. Xenorhinotherium bahiense, MLS 30, dente da série pré-molo-molar superior em vista oclusal; F. Hippidion sp., MLS 50, dente da série pré-molo-molar superior em vista oclusal; G. Palaeolama major, MLS 42, astrágalo direito em vista dorsal. Figuras A, B, E e F modificadas de Santos et al. (2002a).

Figure 3. A. Panochthus greslebini, MLS 13, carapace scute fragment; B. Glyptodon sp., MLS 18, carapace scute; C. Holmesina paulacoutoi, MLS 22, carapace scute; D. Tolypeutes tricinctus, MLS 20, carapace scute; E. Xenorhinotherium bahiense, MLS 30, isolated teeth from the lower molariform series in occlusal view; F. Hippidion sp., MLS 50, isolated teeth from the upper molariform series in occlusal view; G. Palaeolama major, MLS 42, right astragalus in dorsal view. Figures A, B, E e F modified from Santos et al. (2002a). 
Superfamília DASYPODOIDEA Gray, 1821

Família DASYPODIDAE Gray, 1821

Subfamília TOLYPEUTINAE Gray, 1865

Tolypeutes tricinctus (Linnaeus) Gray, 1865

(Figura 3D)

Material. MLS 20-21, osteodermos de carapaça.

Comentários. O gênero Tolypeutes é conhecido desde o Plioceno (Scillato-Yanné, 1980), tendo como representantes atuais Tolypeutes tricinctus e T. matacus. A primeira espécie é encontrada em ambientes de cerrado e caatinga no Brasil (Cartelle, 1999; Eisenberg \& Redford, 1999) e a segunda é mais característica do sul do continente (Wetzel, 1985; Cartelle, 1992). Fósseis relacionados ao gênero no Nordeste do Brasil foram registrados na Bahia (Cartelle, 1992) e, possivelmente, no Piauí (Guérin et al., 1993). No Rio Grande do Norte, uma ocorrência isolada é citada para o município de Rui Barbosa (Porpino et al., 1998). O osteodermo MLS 20, melhor conservado, possui perímetro pentagonal, apresentando superfície externa ornamentada pela presença de tubérculos subarredondados, coincidindo com a descrição de Cartelle (1992) para $T$. tricinctus.

Holmesina paulacoutoi Cartelle \& Bohórquez, 1985 (Figura 3C)

Material. MLS 22-24, osteodermos de carapaça.

Comentários. Alguns autores (Edmund, 1987; Vizcaíno et al., 1998; McKenna \& Bell, 1997; Ranzy, 2000) classificam os gêneros Pampatherium e Holmesina, na família Pampatheriidae, separando-os dos demais Dasypodidae. Cartelle \& Bohórquez (1985) e Cartelle et al. (1989), no entanto, consideram injustificável a referida proposta e agrupar ambos os gêneros na subfamília Pampatheriinae. Paula-Couto (1979) e Guérin et al. (1993) também adotam a classificação na categoria subfamília. Em recente proposta filogenética, Abrantes \& Bergqvist (2003) reposicionam os pampatérios dentro de Dasypodidae, em uma nova tribo da subfamília Tolypeutinae. Restos de Holmesina paulacoutoi Cartelle \& Bohórquez, 1985 foram assinalados para o Rio Grande do Norte nas localidades de Lagoa do Santo, município de Currais Novos (Santos et al., 1990) e Lágea Formosa, município de São Rafael (Oliveira \& Santos, 1990). Os osteodermos apresentam saliência central conspícua e figura central com perfurações mais abundantes e marcadas do que Pampatherium humboldti (Lund) Ameghino, 1875, concordando com as descrições de Cartelle (1992), o que permite atribuí-los a H. paulacoutoi.

Ordem PILOSA Flower, 1883 Superfamília MEGATHERIOIDEA Gray, 1821 Família MEGATHERIIDAE Gray, 1821
Subfamília MEGATHERIINAE Gray, 1821

Eremotherium laurillardi (Lund, 1842)

Material. MLS 25-29, fragmentos de molariformes. Comentários. Com relação à ocorrência do gênero Eremotherium no Quaternário brasileiro, muitas espécies foram criadas com base apenas na proveniência geográfica, e estabelecidas mediante diagnoses fundamentadas em diferenças qualitativas e quantitativas de pouca expressão, conforme apontam Cartelle \& Iullis (1995). No Rio Grande do Norte, E. laurillardi foi registrada em todos os depósitos prospectados, representada nos tanques, principalmente, por material pós-craniano diverso e dentes isolados e fragmentados (vide Oliveira et al., 1989; Santos et al., 1989; Porpino et al., 1998; Santos, 2001), ocorrendo também fragmentos de crânio e dentário nas grutas do Lajedo da Escada, município de Baraúna.

\section{Ordem LITOPTERNA Ameghino, 1889 \\ Subordem LOPHOLIPTERNA Cifelli, 1983 \\ Família MACRAUCHENIIDAE Gervais, 1855 \\ Subfamília MACRAUCHENIINAE Gervais, 1855}

\section{Xenorhinotherium bahiense Cartelle \& Lessa, 1988} (Figura 3E)

Material. MLS 30, dente da série pré-molo-molar inferior. Comentários. Por longo tempo todos os achados brasileiros pertencentes à família Macraucheniidae foram atribuídos a Macrauchenia patachonica Owen, 1838. Cartelle \& Lessa (1988), com base em relevante material coletado no Estado da Bahia, observaram diferenças consideráveis para a proposição de X. bahiense. De acordo com Lessa (1992), esta espécie é característica do Brasil intertropical, enquanto M. patachonica ocorre no Uruguai, Argentina e, possivelmente, sul do Brasil. Entretanto, Guérin et al. (1993) atribuem as descobertas no Pleistoceno do Piauí a $M$. patachonica. No Rio Grande do Norte, a nova espécie já foi assinalada nos municípios de São Rafael (Oliveira et al., 1989), Currais Novos (Porpino \& Santos, 1997), Rui Barbosa e Antônio Martins (Santos, 2001). O dente inferior da série pré-molo-molar estudado é facilmente reconhecível pela marcada selenodontia (Cartelle \& Lessa, 1988) e dimensões, porém o estado fragmentário da superfície do mesmo impede uma descrição mais detalhada.

\section{Ordem NOTOUNGULATA Roth, 1903 \\ Subordem TOXODONTIA Owen, 1853 \\ Família TOXODONTIDAE Owen, 1845}

Material. MLS 31-35, fragmentos de dente.

Comentários. Os achados de Toxodontidae no Rio Grande do Norte foram referidos a Toxodon Owen, 1840 (Souza-Cunha, 1966; Oliveira et al., 1989) e Toxodon platensis Owen, 1840 (Damasceno, 1973; Santos et al., 1989). Neste trabalho, 
apesar da família ser reconhecida com base na disposição das faixas de esmalte, não foi possível, em decorrência da má preservação do material, uma identificação mais precisa, uma vez que a mesma é feita, essencialmente, com base na morfologia dos dentes.

\section{Ordem ARTIODACTYLA Owen, 1848 \\ Subordem TYLOPODA Illiger, 1811 \\ Família CAMELIDAE Gray, 1821 \\ Subfamília CAMELINAE Gray, 1821}

\section{Palaeolama major Liais, 1872}

(Figura 3G)

Material. MLS 36-38, fragmentos de dente; MLS 3940, escafóides; MLS 41 pisiforme; MLS 42-43, astrágalos direitos; MLS 44-45, astrágalos esquerdos; MLS 46, cubóide; MLS 47, falange proximal do membro posterior; MLS 48, falange média do membro posterior.

Comentários. A primeira ocorrência de Camelidae fóssil no Rio Grande do Norte foi registrada por Souza-Cunha (1966), com base em material coletado na caverna Olho d'Água da Escada, atribuído ao gênero Palaeolama. Achados posteriores permitiram o reconhecimento da espécie $P$. major (Oliveira, 1990; Porpino \& Santos, 1997). No Brasil, o táxon é registrado do Rio Grande do Sul ao Nordeste (Cartelle, 1992). Os fragmentos de dente e elementos pós-cranianos estudados no presente trabalho apresentam morfologia e dimensões concordantes com o material descrito e figurado para a espécie por Oliveira (1990) e Cartelle (1992).

\section{Ordem PERISSODACTYLA Owen, 1848 \\ Superfamília EQUOIDEA Hay, 1902 \\ Família EQUIDAE Gray, 1821}

Subfamília EQUINAE Steinmann \& Doderlein, 1890

Equus (Amerhippus) cf. neogaeus Lund, 1840

Material. MLS 49, $\mathrm{P}_{3}$ direito.

\section{Hippidion Owen, 1869}

(Figura 3F)

Material. MLS 50-52, dentes da série pré-molar superior. Comentários. Os primeiros equídeos fósseis no Rio Grande do Norte foram registrados por Souza-Cunha (1966) nos tanques da Fazenda Lágea Formosa, em São Rafael, e atribuídos aos gêneros Equus e Hippidion. Em recente revisão dos achados de Equus (Amerhippus) e Hippidion no Brasil, Alberdi et al. (2003) reconhecem a ocorrência de três espécies: Equus (Amerhippus) neogaeus Lund 1840, Hippidion principale (Lund, 1846) e Hippidion devillei (Gervais, 1855). No material estudado, as peças MLS 50-52 são atribuídas a Hippidion sp. em função de características diagnósticas como a presença de protocone de contorno semicircular e parástilo e mesóstilo bem desenvolvidos (Alberdi et al. 2003; Ubilla \& Alberdi, 1990). A identificação dos dentes individuais da série pré-molo-molar superior é insegura quando baseada em dentes isolados (Cartelle, 1992), razão pela qual são referidos aqui de modo generalizado. O dente MLS 49 é atribuído a E. (Amerhippus) cf. neogaeus com base na semelhança morfológica das cristas do esmalte da superfície oclusal quando comparado aos homólogos figurados em Bergqvist (1989) e Cartelle (1992) para aquela espécie. Por fim, deve-se destacar que uma atribuição específica segura para materiais de Hippidion e Equus (Amerhippus) requer, além dos dentes, o estudo de características cranianas e pós-cranianas, especialmente a análise multivariada dos metapodiais, o que exigiria achados mais completos, em número e diversidade de peças, conforme exemplificado por Alberdi et al. (2003).

\section{DISCUSSÃO}

A associação faunística do Lajedo de Soledade, em comparação com outros tipos de depósitos do Quaternário potiguar, com exceção das cavernas, apresenta maior diversidade taxonômica. Nela estão incluídos taxa sem representação nos tanques até então estudados, como Cerdocyon thous e Protocyon troglodites. Contrasta também com os tanques a predominância de ossos pequenos. Estas características podem refletir muito mais a atuação de processos tafonômicos diferentes em cada jazigo (vide Santos et al., 2002b) e utilização de métodos de coleta distintos, do que diferenças reais nas biocenoces pretéritas associadas aos depósitos.

Do ponto de vista paleoecológico algumas observações podem ser feitas em relação aos taxa identificados. Os pampatérios são comumente considerados como herbívoros ao invés de onívoros ou insetívoros como a maioria dos Dasypodidae (Engelmann, 1985). Além disso, análises morfofuncionais e morfométricas sugerem que os pampatérios, assim como os gliptodontes (principalmente as formas de grande porte como Glyptodon e Panochthus), seriam pastadores, havendo entre eles graus diversos de adaptação a este tipo de hábito alimentar (Vizcaíno, 2000). Além destes, E. laurillardi, P. major, X. bahiense, e E. (A.) cf. neogaeus são também considerados como megamamíferos pastadores. Com relação a Hippidion, a maior robustez das espécies deste gênero, as características morfológicas da região naso-maxilar e uma dentição relativamente braquiodonte, com cristas de esmalte oclusal de menor complexidade que em Equus (Amerhippus),indicariam adaptação para ambientes mais fechados e dieta com pouco material silicoso (Alberdi et al., 2003), classificada por MacFadden \& Shockey (1997) como mista (mixed feeder).

Os taxa associados à megafauna do Lajedo de Soledade compreendem animais com características ecológicas diversas. Leopardus cf. L. tigrinus e C. thous são Carnivora com 
ampla distribuição geográfica, o primeiro ocorrendo preferencialmente em áreas florestais e o segundo em uma apreciável variedade de habitats, desde florestas úmidas até formações abertas (Eisenberg \& Redfod, 1999; Berta, 1982), o que o torna um indicador paleoambiental impreciso. P. troglodites é considerado caçador de campo aberto, cuja subsistência estaria associada à caça de mamíferos de médio porte (Cartelle \& Langguth, 1999). Segundo Trajano \& Ferrarezi (1994), a ocorrência de Ursidae no Quaternário do Nordeste brasileiro é indicativa da vigência de paleoclima mais frio que o atual. A presença de $T$. tricinctus, ocorrente tanto na caatinga quanto no cerrado (Eisenberg \& Redford, 1999), é pouco diagnóstica em termos paleoecológicos, considerando o contexto tratado.

Em seu conjunto, os taxa analisados, particularmente os megaherbívoros e as formas de origem provavelmente austral como P. major e Arctotherium, contrastam com as condições ambientais prevalecentes na região do Lajedo de Soledade, onde atualmente predomina a caatinga hiperxerófila, com abundância de cactáceas e plantas de porte mais baixo e espalhadas, existentes sob temperaturas médias anuais bastante elevadas e baixos índices pluviométricos (Idema, 1999). Por outro lado, estas ocorrências são congruentes com hipóteses que sustentam a existência de cobertura vegetal do tipo savana e temperaturas médias mais baixas para o Pleistoceno final-Holoceno potiguar, como proposto por Mabesoone et al. (1990), concordando com o modelo formulado por Cartelle (1999) para o Brasil Intertropical como um todo. A presença de Leopardus cf. L. tigrinus e Hippidion sp. sugere a coexistência de formações vegetais mais fechadas em associação com as áreas abertas de pastagem. Diante destas considerações, é possível sugerir que o ambiente seria, provavelmente, semelhante àquele inferido por Cartelle (1992) para a Toca dos Ossos, município de Ourolândia, Bahia e à área de São Raimundo Nonato, Piauí, segundo as conclusões de Guérin et al. (1993). As ravinas, como os tanques, representariam locais de confluência da fauna local durante os períodos de seca, por se converterem em reservatórios d'água temporários.

A extinção dos grandes mamíferos, em função das mudanças ambientais que se verificaram em todo o Brasil intertropical durante o Pleistoceno final-Holoceno (Cartelle, 1992), e o possível decréscimo populacional de outras espécies de médio porte em decorrência do mesmo processo são possíveis fatores envolvidos no desaparecimento, na região, de predadores como $S$. populator e $P$. troglodites.

\section{CONCLUSÕES}

É registrada, pela primeira vez, a ocorrência de Arctotherium sp., C. thous, P. troglodites, Leopardus cf. L. tigrinus e T. tricinctus em associação com a megafauna no Estado do Rio Grande do Norte.

O conjunto faunístico analisado sugere que as condições ambientais da região do Lajedo de Soledade durante o
Pleistoceno final-Holoceno eram distintas das atuais, concordantes com a proposta de Mabesoone et al. (1990) e provavelmente semelhantes àquelas inferidas para regiões da Bahia e Piauí, durante o mesmo período, o que compreenderia áreas abertas de savana associadas, possivelmente, com fisionomias mais fechadas

\section{AGRADECIMENTOS}

A Alfredo Langguth (UFPB), pela gentileza em possibilitar o exame da coleção osteológica de mamíferos do Laboratório de Citogenética; a Leonardo Menezes, pelas fotografias originais; a Francisco P. de Lima Filho (UFRN), pelo empréstimo do equipamento fotográfico; a Leopoldo $\mathrm{H}$. Soilbelzon e Sergio F. Vizcaíno (MLP, Argentina) pelo envio de material bibliográfico; a Ana Maria Ribeiro e ao revisor anônimo pelas valiosas sugestões que muito contribuíram para o aprimoramento deste trabalho.

\section{REFERÊNCIAS}

Abrantes, E.A.L. \& Bergqvist, L.P. 2003. Proposta filogenética para os Dasypodidae (Mammalia: Cingulata). In: SIMPÓSIO BRASILEIRO DE PALEONTOLOGIA DE VERTEBRADOS, 3, 2003. Boletim de Resumos, Rio de Janeiro, UERJ, p. 11.

Alberdi, M.T.; Cartelle, C. \& Prado, J.L. 2003. El registro Pleistoceno de Equus(Amerhippus) e Hippidion (Mammalia, Perissodactyla) de Brasil. Consideraciones paleoecológicas y biogeográficas. Ameghiniana, 40(2):173-196.

Bagnoli, E. 1994. O Lajedo de Soledade, Apodi (RN) um exemplo de preservação do patrimônio cultural brasileiro. Revista de Arqueologia, 8(1):239-253.

Bergqvist, L.P. 1989. Os mamíferos pleistocênicos do Estado da Paraíba, Brasil, depositados no Museu Nacional, Rio de Janeiro. Programa de Pós-graduação em Zoologia, Museu Nacional, Universidade Federal do Rio de Janeiro, Dissertação de Mestrado, $174 \mathrm{p}$.

Bergqvist, L.P. 1995. Jazimentos pleistocênicos do Estado da Paraíba e seus fósseis. Revista Nordestina de Biologia, 8(2):143-158.

Berta, A. 1982. Cerdocyon thous. Mammalian Species, 186:1-4.

Born, P.A. \& Sedor, F.A. 2001. Ocorrência de Protocyon troglodites (Canidae, Carnivora) e de Cervidae (Artyodactyla) no Pleistoceno do Estado do Paraná. In: CONGRESSO BRASILEIRO DE PALEONTOLOGIA, 17, 2001. Resumos, Rio Branco, UFAC, p.178.

Cartelle, C. 1992. Edentata e megamamíferos herbívoros extintos da Toca dos Ossos (Ourolândia, BA, Brasil).Programa de PósGraduação em Morfologia, Universidade Federal de Minas Gerais, Tese de Doutorado, 516 p.

Cartelle, C. 1994. Tempo passado. Mamíferos do Pleistoceno em Minas Gerais. Belo Horizonte, Editora palco, 131 p.

Cartelle, C. 1998. Um pequeno urso do Pleistoceno final da Bahia. Acta Geológica Leopoldensia, 21(46/47):171-189.

Cartelle, C. 1999. Pleistocene Mammals of the Cerrado and Caatinga of Brazil. In: J.F Eisenberg. \& K.H. Redford (eds.) Mammals of the Neotropics. The central Tropics. 3, The University of Chicago Press, p. 27-46.

Cartelle, C. \& Bohórquez, G.A. 1985. Pampatherium paulacoutoi, uma nova espécie de tatu gigante da Bahia, Brasil (Edentata, 
Dasypodidae). Revista Brasileira de Biologia, 2(4):229-254.

Cartelle, C. \& Lessa, G. 1988. Descrição de um novo gênero e espécie de Macraucheniidae (Mammalia, Litopterna) do Pleistoceno do Brasil. Paulacoutiana, 3:3-26.

Cartelle, C.\& Abuhid, V.S., 1989. Novos espécimes de Smilodon populator (Lund, 1842), Carnívora, Machairodontinae: morfologia e conclusões taxonômicas. In: CONGRESSO BRASILEIRO DE PALEONTOLOGIA, 11, 1989. Anais, Curitiba, UFPR, p. 607-620.

Cartelle, C.; Câmara, B.G. \& Prado, P.I.L. 1989. Estudo comparativo dos esqueletos da mão e pé de Pampatherium humboldti (Lund, 1839) e Holmesina paulacoutoi (Cartelle \& Bohórquez, 1985) (Edentata, Pampatheriinae). In: CONGRESSO BRASILEIRO DE PALEONTOLOGIA, 11, 1989. Anais, Curitiba, UFPR, p. 621-634.

Cartelle, C. \& Iuliis, G. 1995. Eremotherium laurillardi: the panamerican Late Pleistocene megatheriid sloth. Journal of Vertebrate Paleontology, 15(4):830-841.

Cartelle, C. \& Hartwig, W.C. 1996. A new extinct primate from the pleistocene megafauna of Bahia, Brazil. Proceedings of National Academy of Science, 93:6405-6409.

Cartelle, C. \& Langguth, A. 1999. Protocyon troglodites (Lund): um canídeo intertropical extinto. Anais da Academia Brasileira de Ciências, 71(3-I):371-384.

Córdoba, V.C.; Spadini, A.R. \& Bagnoli, E. 1994. Lajedo de Soledade, Cretáceo Superior da bacia Potiguar: um exemplo de sedimentação carbonática, sob influência de marés. In: CONGRESSO BRASILEIRO DE GEOLOGIA, 36, 1994. Anais, Camboriú, SBG, p. 293-295.

Damasceno, J.M. 1973. Ocorrência de Toxodon platensis Owen, 1840 em Olho d'água da Escada, Município de Mossoró, Rio Grande do Norte. Arquivos do Instituto de Antropologia 1:1-18.

Edmund, A.G. 1987. Evolution of the genus Holmesina (Pampatheriidae, Mammalia) in Florida, with remarks on taxonomy and distribution. Pearce-Sellards Series 45:1-18.

Eisenberg, J.F. \& Redford, K.H.1999. Mammals of the Neotropics. The central Neotropics. 3. The University of Chicago Press, $609 \mathrm{p}$.

Engelmann, F.F. 1985. The phylogeny of the Xenarthra. In: Montgomery, G.G. (ed) The evolution and ecology of armadillos, sloths and vermilinguas, Smithsonian Institution Press, p. 51-64.

Gomide, M. 1989. Mamíferos pleistocênicos de Itapipoca, Ceará, Brasil, depositados no Museu Nacional. Programa de Pós-graduação em Zoologia, Museu Nacional, Universidade Federal do Rio de Janeiro, Dissertação de Mestrado, 172 p.

Gomide, M.; Bergqvist, L.P. \& Rêgo, D.D. 1987. O "tigre-dentede-sabre" (Smilodon populator), de Itapipoca, Ceará. In: CONGRESSO BRASILEIRO DE PALEONTOLOGIA, 10, 1987. Anais, Rio de Janeiro, UFRJ, p. 197-207.

Guérin, C.; Hugueney, M.; Mourer-Chauviré, C. \& Faure, M. 1993. Paléoenvironement pléistocène dans l'aire archéologique de São Raimundo Nonato (Piaui, Brésil): apport des mammifères et des oiseaux. Documentation du Laboratoire de Géologie de Lyon, 125:187-202.

IDEMA. 1999. Apodi. Informativo Municipal, 5:1-14.

Lessa, G.M. 1992. Estudo descritivo de Xenorhinotherium bahiense Cartelle \& Lessa, 1988 e comparação com outras espécies de Macraucheniidae (Litopterna, Mammalia). Programa de PósGraduação em Geologia, Universidade Federal do Rio de Janei- ro, Dissertação de Mestrado, 264 p.

Lessa, G.; Cartelle, C.; Faria, H.D. \& Gonçalves, P.R. 1999. Novos achados de mamíferos carnívoros do Pleistoceno final-Holoceno em grutas calcárias do Estado da Bahia. Acta Geologica Leopoldensia, 21(46/47):157-169.

Mabesoone, J.M.; Oliveira, L.D.D. \& Damasceno, J.M. 1990. Desenvolvimento dos tanques fossilíferos no Semi-Árido Norteriograndense. In: CONGRESSO BRASILEIRO DE GEOLOGIA, 36, 1990. Anais, Natal, UFRN, p. 733-741.

MacFadden, B.J. \& Shockey, B.J. 1997. Ancient feeding ecology and niche differentiation of Pleistocene mammalian herbivores from Tarija, Bolivia: morphological and isotopic evidence. Paleobiology, 23(1):77-100.

McKenna, M.C \& Bell, S.K. 1997. Classification of mammals above the species level. Columbia University Press, New York, 631 p.

Moreira, L.E. 1971. Os gliptodontes do nordeste do Brasil. Anais da Academia Brasileira de Ciências, 43(suplemento): 529-552.

Nowak, R.M. 1991. Walker's mammals of the world. Vol. II. $5^{\mathrm{a}}$ ed.. The John Hopkins University Press, 1629 p.

Oliveira, E.V. 1996. Mamíferos Xenarthra (Edentata) do Quaternário do Estado do Rio Grande do Sul. Ameghiniana, 33(1): 65-75.

Oliveira, L.D.D. 1990. Importância da ocorrência de Palaeolama major (Liais, 1872) na Fazenda Lájea Formosa, São Rafael-RN (Mammalia, Camelidae). In: CONGRESSO BRASILEIRO DE GEOLOGIA, 36, 1990. Anais, Natal, UFRN, p. 506-513.

Oliveira, L.D.D.; Santos, C.L.A.\& Santos, M.F.C.F. 1982. Primeira ocorrência de Parapanochthus Moreira, 1965, no Rio Grande do Norte. Boletim do Departamento de Geologia CCE/UFRN, 4:19-22

Oliveira, L.D.D.; Santos, C.L.A.\& Santos, M.F.C.F. 1984. Nova ocorrência de Panochthus greslebini Castellanos, 1941, no Estado do Rio Grande do Norte. In: SIMPÓSIO DE GEOLOGIA DO NORDESTE, 1, 1989. Resumos, Natal, UFRN, p. 346-351.

Oliveira, L.D.D.; Damasceno, J.M.; Lins, F.A.P.; Walter, E.M. \& Moreira, J.A. 1989. Estudo macrofossilífero dos tanques da Fazenda Capim Grosso, São Rafael-RN, auxiliado por métodos geofísicos. In: CONGRESSO BRASILEIRO DE PALEONTOLOGIA, 11, 1989. Anais, Curitiba, UFPR, p. 551570.

Oliveira, L.D.D. \& Santos, M.F.C.F. 1990. Uma provável ocorrência de Holmesina paulacoutoi (Cartelle \& Bohórquez, 1985) nos tanques fossilíferos de Lágea Formosa, São Rafael-RN. Caatinga 7(único):164-166.

Paiva, M.P. \& Campos, E. 1995. Fauna do Nordeste do Brasil. Fortaleza, Banco do Nordeste, $274 \mathrm{p}$.

Paula-Couto, C. 1960. Um urso extinto do Brasil. Boletim da Sociedade Brasileira de Geologia, 9(1):5-27.

Paula-Couto, C. 1979. Tratado de Paleomastozoologia.Rio de Janeiro, Academia Brasileira de Ciências, 590 p.

Paula-Couto, C. 1983. Fossil mammals from the Cenozoic of Acre, Brazil VI-Edentata Cingulata. Iheringia, Série Geológica, 8:33-49.

Porpino, K.O. 2000. Sobre a ocorrência de Glyptodon clavipes Owen, 1839 e Glyptodon reticulatus Owen, 1845 no Estado do Rio Grande do Norte. In: SIMPÓSIO BRASILEIRO DE PALEONTOLOGIA DE VERTEBRADOS, 2, 2000. Resumos, Rio de Janeiro, Museu Nacional/UFRJ, p. 48.

Porpino, K.O. \& Bergqvist, L.P. 2002. Novos achados de Panochthus (Mammalia, Cingulata, Glyptodontoidea) no Nordeste do Brasil. Revista Brasileira de Paleontologia, 4:51-62.

Porpino, K.O. \& Santos, M.F.C.F. 1997. Mamíferos pleistocênicos 
de Lagoa do Santo, Rio Grande do Norte-Brasil. In: CONGRESSO BRASILEIRO DE PALEONTOLOGIA, 15, 1997. Resumos, São Pedro,UNESP, p. 116.

Porpino, K.P., Santos, M.F.C.F., Carvalho-Neto, A. T., 1998. Investigações paleontológicas no jazigo fossilífero da Fazenda Acauã, Rui Barbosa-RN. In: REUNIÃO ANUAL DA SBPC, 50,1998. Resumos, Natal, UFRN/SBPC, p. 1035.

Ranzy, A. 2000. Paleoecologia da Amazônia - Megafauna do Pleistoceno. Florianópolis, Editora da UFSC, 101 p.

Ribeiro, A.M.; Rodrigues, P.H. \& Ferigolo, J. 2003. Primeiro registro de Ursidae (Tremarctinae) para o Pleistoceno do Estado do Rio Grande do Sul, Brasil. In: JORNADAS ARGENTINAS DE PALEONTOLOGIA DE VERTEBRADOS, 19, 2003. Resúmenes, Buenos Aires, p. 27.

Rosado, V. 1957. A Formação Cacimbas e o Grupo Apodi. Coleção Mossoroense, 24:1-21.

Santos, M.F.C.F. 2001. Geologia e paleontologia de depósitos fossilíferos pleistocênicos do Rio Grande do Norte. Programa de Pós-Graduação em Geodinâmica e Geofísica, Universidade Federal do Rio Grande do Norte, Dissertação de Mestrado, $70 \mathrm{p}$.

Santos, M.F.C.F.; Oliveira, L.D.D. \& Santos, C.L.A. 1989. Mamíferos quaternários da Lagoa de Lajes, Alexandria, RN. Ordens: Edentata, Proboscidea e Notoungulata. In: CONGRESSO BRASILEIRO DE PALEONTOLOGIA, 11, 1989. Anais, Curitiba, UFPR, p. 689-710.

Santos, M.F.C.F.; Oliveira, L.D.D. \& Santos, C.L.A. 1990. Considerações sobre a primeira ocorrência de Pampatherium paulacoutoi Cartelle \& Bohórquez, 1985, no Rio Grande do Norte. In: CONGRESSO BRASILEIRO DE GEOLOGIA, 36, 1990. Anais, Natal, UFRN, p. 503-505.

Santos, M.F.C.F.; Lima-Filho, F.P. \& Bergqvist, L.P. 2002a. Fósseis pleistocênicos da Ravina do Leon, Lajedo de Soledade, Apodi/RN. Revista de Geologia, 15:23-29.

Santos, M.F.C.F.; Bergqvist, L.P.; Lima-Filho, F.P. \& Pereira, M.M.V. 2002b. Feições tafonômicas observadas em fósseis pleistocênicos do Rio Grande do Norte. Revista de Geologia, 15:31-41.

Scillato-Yanné, G.J. 1980. Los Xenarthra fósiles de Argentina (Mammalia, Edentata). In: CONGRESO ARGENTINO DE PALEONTOLOGÍA Y BIOESTRATIGRAFÍA, 4, 1986. Actas, Mendonza, p. 151-155.

Soibelzon, L.H. 2004. Revisión sistemática de los Tremarctinae (Carnivora, Ursidae) fósiles de América del Sur. Revista del Museo Argentino de Ciencias Naturales, 6(1):107-133.

Souza-Cunha, F.L. 1966. Explorações paleontológicas no Pleistoceno do Rio Grande do Norte. Arquivos do Instituto de Antropologia, 2(1-2):75-116.

Trajano, E. \& Ferrarezzi, H. 1994. A fossil bear from northeastern Brazil with a phylogenetic analysis of the South American extinct Tremarctinae (Ursidae). Journal of Vertebrate Paleontology, 14(4):552-561.

Ubilla, M. \& Alberdi, M.T. 1990. Hippidion sp. (Mammalia, Perissodactyla, Equidae) en sedimentos del Pleistoceno Superior del Uruguay (Edad Mamifero Lujanense). Estudios Geológicos, 46:453-464.

Vizcaíno, S.F. 2000. Vegetation Partitioning among Lujanian (LatePleistocene/Early Holocene) armored herbivores in the Pampean Region. Current Research in the Pleistocene, 17:135-137.

Vizcaíno, S.F.; De Iuliis, G.\& Bargo M.S. 1998. Skull shape, masticatory apparatus, and diet of Vassalia and Holmesina (Mammalia: Xenarthra: Pampatheriidae): when anatomy constrains destiny. Journal of Mammalian Evolution, 5(4):291-322.

Wetzel, R.M. 1985. Taxonomy and distribution of armadillos, Dasypodidae. In: G.G. Montgomery, (ed.) The evolution and ecology of armadillos, sloths, and vermilinguas, Smithsonian Institution Press, p. 23-50.

Winge, H. 1915. Jordfundne og nulevende Gumlere (Edentata) fra Lagoa Santa, Minas Geraes, Brasilien. E Museo Lundi, 3(2):1-321.

Received February, 2004; accepted August, 2004. 